\title{
Extended Coulomb liquid of paired hardcore boson model on a pyrochlore lattice
}

\author{
Chun-Jiong Huang $\odot,{ }^{1,2,3,4}$ Changle Liu, ${ }^{5,6}$ Ziyang Meng, ${ }^{7}$ Yue Yu, ${ }^{5,6,9}$ Youjin Deng $\odot,{ }^{2,3,4,{ }^{*}}$ and Gang Chen $\circledast^{1,5,6,8,9, \dagger}$ \\ ${ }^{1}$ Department of Physics and HKU-UCAS Joint Institute for Theoretical and Computational Physics at Hong Kong, \\ University of Hong Kong, Hong Kong, China \\ ${ }^{2}$ Shanghai Branch, National Laboratory for Physical Sciences at Microscale and Department of Modern Physics, \\ University of Science and Technology of China, Shanghai, 201315, China \\ ${ }^{3}$ CAS Center for Excellence and Synergetic Innovation Center in Quantum Information and Quantum Physics, \\ University of Science and Technology of China, Hefei, Anhui 230026, China \\ ${ }^{4}$ CAS-Alibaba Quantum Computing Laboratory, Shanghai, 201315, China \\ ${ }^{5}$ State Key Laboratory of Surface Physics and Department of Physics, Fudan University, Shanghai, 200433, China \\ ${ }^{6}$ Center for Field Theory and Particle Physics, Fudan University, Shanghai, 200433, China \\ ${ }^{7}$ Beijing National Laboratory for Condensed Matter Physics and Institute of Physics, CAS, Beijing 100190, China \\ ${ }^{8}$ Institute for Nanoelectronic Devices and Quantum Computing, Fudan University, Shanghai, 200433, China \\ ${ }^{9}$ Collaborative Innovation Center of Advanced Microstructures, Nanjing University, Nanjing, 210093, China
}

(Received 27 June 2018; revised 17 October 2020; accepted 20 October 2020; published 30 October 2020)

\begin{abstract}
There is growing interest in the $U(1)$ Coulomb liquid in both quantum materials in pyrochlore ice and cluster Mott insulators and cold-atom systems. We explore a paired hardcore boson model on a pyrochlore lattice. This model is equivalent to the XYZ spin model that was proposed for rare-earth-metal pyrochlores with "dipoleoctupole" doublets. Since our model has no sign problem for quantum Monte Carlo (QMC) simulations in a large parameter regime, we carry out both analytical and QMC calculations. We find that the $U(1)$ Coulomb liquid is quite stable and spans a rather large portion of the phase diagram with boson pairing. Moreover, we numerically find a thermodynamic evidence that the boson pairing could induce a possible $\mathbb{Z}_{2}$ liquid in the vicinity of the phase boundary between Coulomb liquid and $\mathbb{Z}_{2}$ symmetry-broken phase. Besides the materials' relevance with quantum spin ice, we point to quantum simulation with cold atoms on optical lattices.
\end{abstract}

DOI: 10.1103/PhysRevResearch.2.042022

Introduction. The search of exotic quantum phases with quantum number fractionalization and emergent gauge structure has been an active subject in modern condensed matter physics. One theoretical route in the field is to start from the exotic phase itself and construct solvable models. These models are often contrived and not quite realistic [1-4]. One exception is the exactly solvable honeycomb lattice $\mathrm{Ki}$ taev model [5], whose physical relevance to the iridates was pointed out by Jackeli and Khaliullin [6]. The opposite route is to start from the realistic systems and build up relevant models from the microscopic degrees of freedom. Both routes have been fruitful. The latter route faces several major obstacles. First, constructing a relevant physical model is not often straightforward. Second, these strongly interacting models often cannot be solved in a controlled manner. Occasionally, certain realistic models, such as the square lattice Heisenberg model for cuprates, may be solved but yield mundane and

\footnotetext{
*yjdeng@ustc.edu.cn

†gangchen.physics@gmail.com
}

Published by the American Physical Society under the terms of the Creative Commons Attribution 4.0 International license. Further distribution of this work must maintain attribution to the author(s) and the published article's title, journal citation, and DOI. known results and are thus of limited theoretical value for our understanding of strongly correlated quantum matters. Therefore, a physically relevant model that can be solved in a controlled manner and at the same time gives nontrivial quantum phases is highly valuable in the study of strongly correlated quantum matters.

The XYZ spin model, derived from the microscopics of dipole-octupole doublets on pyrochlore and triangular lattices in Refs. [7,8], is a rare example that overcomes the major obstacles of the second route. It was suggested that this model on the pyrochlore lattice could stabilize a $U(1)$ Coulombic liquid $[9,10]$ and may stabilize a $\mathbb{Z}_{2}$ spin liquid in parts of its phase diagram [7]. The $U(1)$ Coulombic liquid is an exotic quantum state that is described by compact quantum electrodynamics with emergent quasiparticles $[9,10]$ and has found relevance in pyrochlore quantum ice materials [7,11-22] and cluster Mott insulators [23-27]. Besides the nontrivial ground states, it was further pointed out [7] that this model does not have a sign problem for quantum Monte Carlo (QMC) simulation in a large parameter regime, and in fact it is the case for any lattice [8]. An interesting extension of this model to the kagomé lattice by dimensional reduction from the pyrochlores with magnetic fields was pursued numerically in Ref. [28] where a $\mathbb{Z}_{2}$ spin liquid in the kagomé ice regime was suggested. Our model was initially proposed for various Nd-based pyrochlore materials [7,29- 
37] and was recently suggested for Ce-based pyrochlore spin liquid candidates $\mathrm{Ce}_{2} \mathrm{Sn}_{2} \mathrm{O}_{7}$ and $\mathrm{Ce}_{2} \mathrm{Zr}_{2} \mathrm{O}_{7}$ [38-43]. Thus, this model becomes a rare model that describes real physical systems, supports nontrivial quantum phases, and can be solved in a controlled manner in a large parameter regime. Inspired by these compelling properties $[7,8,38]$, we show that the Coulomb liquid covers a rather large portion of the phase diagram. Moreover, the physical boson pairing may render new fates to the emergent spinon-gauge coupling in the Coulomb liquid $[14,15]$. We find the thermodynamic evidence for the possible existence of a $\mathbb{Z}_{2}$ liquid out of the $U(1)$ Coulomb liquid via an internal Anderson-Higgs mechanism by the spinon pairing.

The model. We start from the paired hardcore boson model on the pyrochlore lattice with

$$
H=\sum_{\langle i j\rangle}\left[\left(-t_{1} b_{i}^{\dagger} b_{j}-t_{2} b_{i}^{\dagger} b_{j}^{\dagger}+\text { H.c. }\right)+V n_{i} n_{j}\right] .
$$

Here, $b_{i}^{\dagger}\left(b_{i}\right)$ creates (annihilates) one boson at site $i$, and $n_{i} \equiv b_{i}^{\dagger} b_{i}$ is the boson occupation number. This model differs from the usual hardcore boson model $[9,26,44-46]$ by having an extra boson pairing term. Previous theoretical works and numerical efforts on the hardcore boson model without the boson pairing have established the presence of the Coulomb liquid that supports the gapless $U(1)$ gauge photon and fractionalized excitations [44,47]. Our main purpose is to understand the role of this boson pairing on the phase diagram.

Our model has a strong physical motivation. Via the standard mapping $b_{i} \equiv S_{i}^{-}, n_{i} \equiv S_{i}^{z}+1 / 2$, this model is identical to the XYZ spin model that was derived as a generic and realistic model for the interaction between the so-called "dipole-octupole doublets" on the pyrochlore lattice [7,8,38]. The boson pairing naturally arises from the spin-orbit entanglement of dipole-octupole doublets. At the end of this Rapid Communication, we further mention the relevance with the cold-atom systems $[48,49]$. Because of the boson pairing, the global $U(1)$ symmetry is absent and the total boson number is not conserved, but the Hamiltonian remains invariant under a global $\mathbb{Z}_{2}$ (or Ising) symmetry transformation with $b_{i} \rightarrow-b_{i}, b_{i}^{\dagger} \rightarrow-b_{i}^{\dagger}$. Throughout this Rapid Communication, we work on the regime with an average 1/2-boson filling. In the following, we first carry out the theoretical analysis and provide the physical understanding of the internal and emergent gauge structure and fractionalized excitations of this model and then implement the large-scale QMC simulation to confirm the theoretical expectation.

The internal gauge structure and phase diagram. Since the hardcore boson model without $t_{2}$ pairing is equivalent to the XXZ spin model and has been extensively studied [9,44-46], we briefly explain the ground state in this limit. When the hopping $t_{1}$ exceeds a critical value, the bosons are simply condensed and form a superfluid by breaking the global $U(1)$ symmetry. In the opposite case when $t_{1}$ is less than a critical value, the system would form a $U(1)$ Coulomb liquid with emergent $U(1)$ gauge structure. Note this emergent $U(1)$ gauge structure in the $U(1)$ Coulomb liquid has nothing to do with the global $U$ (1) symmetry of the model in the XXZ limit. Because of the emergent nonlocality of the underlying $U(1)$ gauge structure, the Coulomb liquid in the small- $t_{1}$ regime is robust against any small and local perturbation such as the weak $t_{2}$ boson pairing.

The $U$ (1) Coulomb liquid in the phase diagram can also be established from the limit with $t_{1}=0$. As we elaborate in Ref. [50], a sixth-order degenerate perturbation theory in the $t_{2}$ pairing is needed to generate the three-boson hopping on the perimeter of the elementary hexagons of the pyrochlore lattice. It is this three-boson collective hopping that allows the system to fluctuate quantum mechanically within the extensively degenerate ground-state manifold (or spin ice [9,51-53] manifold in the spin language) of the predominant boson interaction and lead to the Coulomb liquid. When both $t_{1}$ and $t_{2}$ are present and remain small, similar perturbative treatment again leads to Coulomb liquid. Therefore, we expect the Coulomb liquid to appear as the ground state when both $t_{1}$ and $t_{2}$ are reasonably smaller than $V$.

To establish the phase diagram, we first realize that the system favors a ferromagnetic order with $\left\langle S^{x}\right\rangle \equiv\left\langle b+b^{\dagger}\right\rangle / 2 \neq$ 0 when $t_{1}, t_{2} \gg V$ and $t_{1}, t_{2}>0$. Moreover, the phases for $t_{2}>0$ and $t_{2}<0$ are related under the transformation $b_{j} \rightarrow$ $i b_{j}$. To reveal the connection between the Coulomb liquid and the ferromagnet, we view the Coulomb liquid as the parent phase and implement the spinon-gauge construction [14,15] for the hardcore boson operators that is appropriate for the Coulomb liquid phase,

$$
b_{i}^{\dagger} \equiv \frac{1}{2} \Phi_{r}^{\dagger} \Phi_{r^{\prime}} e^{i A_{r^{\prime}}}, \quad \sum_{i \in \mathrm{tet}_{r}} n_{i}=\eta_{r} Q_{r}+2,
$$

where $\Phi_{r}^{\dagger}\left(\Phi_{r}\right)$ creates (annihilates) a spinon at the center (labeled by $\boldsymbol{r}$ ) of the tetrahedron (tet ${ }_{r}$ ), and $\eta_{\boldsymbol{r}}= \pm 1$ for two sublattices of the diamond lattice formed by the tetrahedral centers. As we explain in detail in Ref. [50], the paired hardcore boson model becomes

$$
\begin{aligned}
H= & \sum_{r} \frac{V Q_{r}^{2}}{2}-\frac{t_{1}}{4} \sum_{\left[r r^{\prime}\right]} \Phi_{r}^{\dagger} \Phi_{r^{\prime}} e^{-i\left(A_{r r^{\prime \prime}}+A_{r^{\prime \prime} r^{\prime}}\right)} \\
& -\frac{t_{2}}{8} \sum_{\left\langle\boldsymbol{r} \boldsymbol{r}^{\prime \prime}\right\rangle} \sum_{\left\langle\boldsymbol{r}^{\prime} \boldsymbol{r}^{\prime \prime}\right\rangle}\left[\Phi_{\boldsymbol{r}^{\prime \prime}}^{\dagger} \Phi_{\boldsymbol{r}^{\prime \prime}}^{\dagger} \Phi_{\boldsymbol{r}^{\prime}} \Phi_{\boldsymbol{r}^{\prime \prime}} e^{-i\left(A_{r^{\prime \prime}}+A_{r^{\prime} r^{\prime \prime}}\right)}+\text { H.c. }\right],
\end{aligned}
$$

where $\langle\cdots\rangle([\cdots])$ means the nearest (next-nearest) neighbors and $\boldsymbol{r}^{\prime \prime}$ is the common nearest neighbor of $\boldsymbol{r}$ and $\boldsymbol{r}^{\prime}$. The boson pairing mediates the spinon interaction in the spinon-gauge formulation [14] that may induce pairing between these fractionalized degrees of freedom and thus gap out the continuous part of the internal $U(1)$ gauge field via an internal AndersonHiggs mechanism. Through mean-field analysis, we do not actually find any pairing instability within the Coulomb liquid [see Fig. 1(a)] that can be a mean-field artifact. Nevertheless, the mean-field theory gives a large region for Coulomb liquid in Fig. 1(a).

QMC results for different phases. To examine our understanding, we numerically solve the model in Eq. (1) by performing the the worm-type QMC algorithm [54,55] and a brief description of this algorithm can be found here [50]. We set the chemical potential $\mu \equiv 3 V$ to comply with the $\mathrm{XYZ}$ model. To determine the phase boundary between the liquid phases and the ferromagnet, we monitor the first-order 


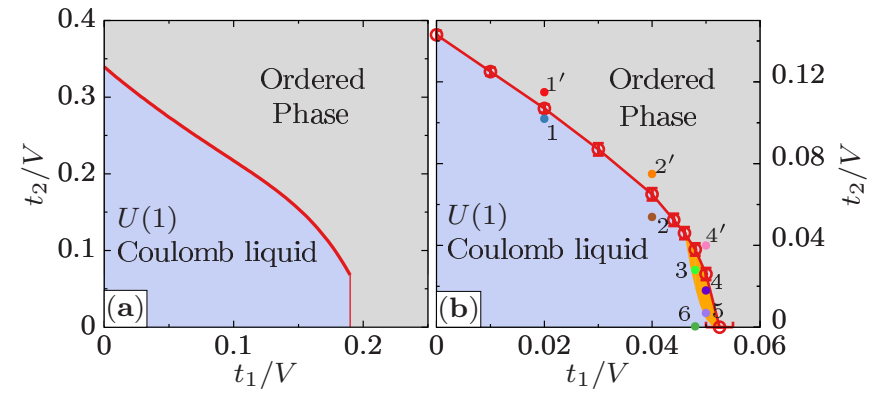

FIG. 1. (a) The mean-field phase diagram. Thick (thin) line indicates a first-order (continuous) transition. (b) The QMC phase diagram with the system size $L=8$ and the temperature $T / V=1 / 800$. The detailed properties of the specific points are presented in Figs. 3 and 4 . The orange region is the candidate $\mathbb{Z}_{2}$ spin liquid phase and the transition from the $U(1)$ Coulomb liquid to the $\mathbb{Z}_{2}$ liquid is continuous.

derivative of the free energy $\ln \mathcal{Z}$ over $t_{1}$ and $t_{2}$ with

$$
\mathcal{E}_{1}=\frac{\partial \ln \mathcal{Z}}{\partial t_{1}}=\frac{\left\langle k_{1}\right\rangle}{t_{1}}, \quad \mathcal{E}_{2}=\frac{\partial \ln \mathcal{Z}}{\partial t_{2}}=\frac{\left\langle k_{2}\right\rangle}{t_{2}} .
$$

We simulate these values by varying $t_{2}$ for fixed $t_{1}$ 's with the system size $N=4 \times 8^{3}, \beta=\left(k_{\mathrm{B}} T\right)^{-1}=800$, where we set $V=1$ as the energy unit. The numerical phase diagram is presented in Fig. 1(b). As we calculate in Fig. 2, the transitions are strongly first order at small $t_{1}$ 's and are consistent with the theoretical results in Fig. 1(a). Moreover, as the system approaches the phase boundary near the horizontal axis, the transition becomes weakly first-order like. In general, the phase boundary in Fig. 1(b) is qualitatively consistent with the theoretical one.

To understand different phases, we probe the thermodynamic properties by measuring the specific heat and entropy

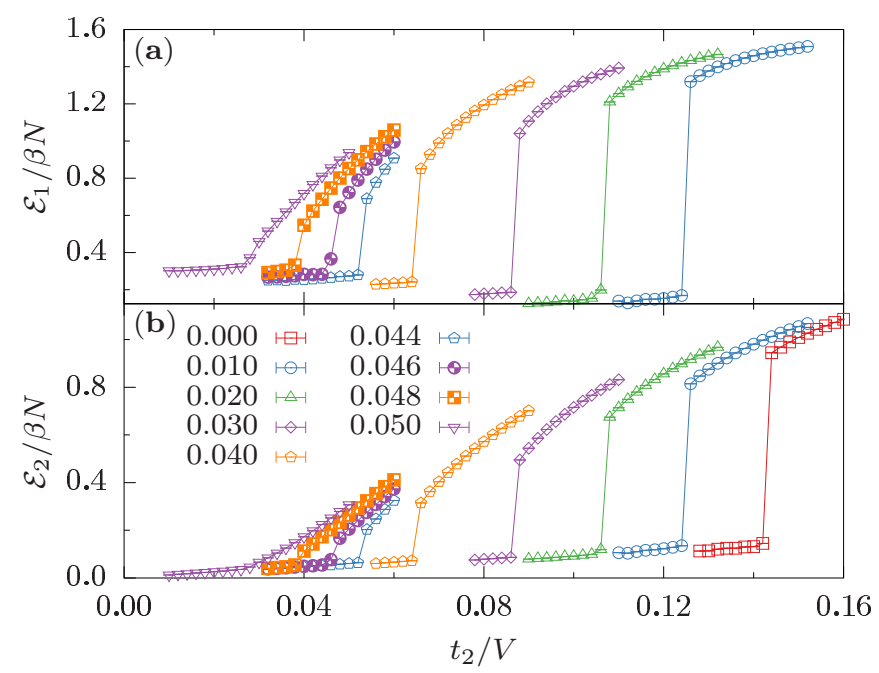

FIG. 2. The hopping and pairing kinks by varying couplings. The legend suggests the values of $t_{1} / V$. The system size is $N=4 \times 8^{3}$ and the temperature $T / V=1 / 800$. When $t_{1} \leqslant 0.048 \mathrm{~V}$ the curves of both kink types are clearly discontinuous, indicating strongly firstorder transitions. For $t_{1}=0.048 \mathrm{~V}, 0.05 \mathrm{~V}$, a weakly first-order phase transition is more likely.

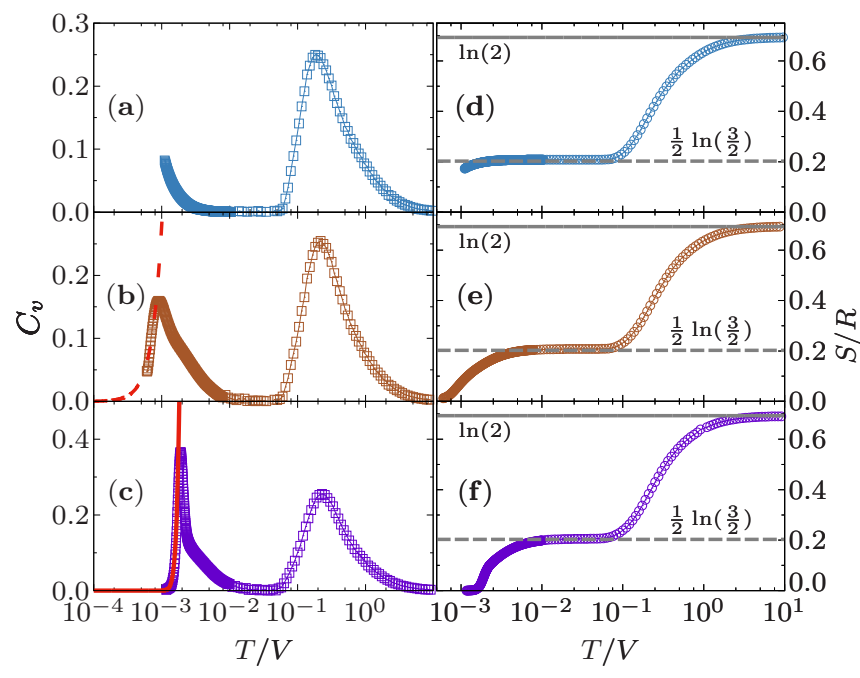

FIG. 3. Panels (a), (b), and (c) are heat capacities for points 1, 2, and 4, respectively. Panels (d), (e), and (f) are entropy densities for points 1,2 , and 4 , respectively. Dashed (solid) curve is a fit of $T^{3}$ (exponential decaying) behavior.

for representative points in Fig. 1(b). The results are depicted in Fig. 3. For the Coulomb liquid in the pyrochlore ice context $[9,10,53]$, it is well known that there exist double peaks in the heat capacity. The high-temperature peak signals the entrance into the spin ice manifold, while the low-temperature peak arises from the quantum fluctuation that breaks the classical degeneracy of the spin ice manifold. Between two peaks, there is an entropy plateau at Pauling entropy since the system is thermally fluctuating within the ice manifold. Below the low-temperature peak, the specific heat behaves as $C_{v} \propto T^{3}$ in the zero-temperature limit due to the gapless gauge photon [15]. For points 1 and 2 in Fig. 1(b), the specific heat behavior is consistent with the Coulomb liquid (see Fig. 3). This gapless excitation is the key signature of the emergent gauge dynamics and is unrelated to any continuous symmetry breaking, especially since there is no symmetry breaking in the disordered regime and our model [7] does not have any continuous symmetry.

For the $\mathbb{Z}_{2}$ liquid, all excitations are gapped. Since the spinon pairing is expected to occur at very low energy scale, the double peaks in heat capacity should persist except that we have an activated behavior of the heat capacity below the low-temperature peak instead of the $T^{3}$ behavior for the Coulomb liquid. Inside the disordered regime of Fig. 1(b), we find that the behaviors of "points $3,4,5$ " are consistent with a $\mathbb{Z}_{2}$ liquid (see Fig. 3 and Ref. [50]). This result provides a thermodynamic evidence for the presence of a $\mathbb{Z}_{2}$ liquid phase in the (orange) region of the disordered regime. More specifically, the thermodynamic gap that is extracted from the heat capacity for the point 4 is $\approx 0.018 \mathrm{~V}$. This is of the same order as $t_{2}$, suggesting the physical origin of the $\mathbb{Z}_{2}$ liquid state. As was noted, the $t_{2}$ term renders an effective interaction between the (fractionalized) spinon quasiparticles. When one pair of spinons is condensed and individual spinon remains uncondensed, the Coulomb liquid would give way to the $\mathbb{Z}_{2}$ liquid in a way similar to the superconducting pairing transition in a BCS superconductor. More physically, 
TABLE I. The physical properties of different phases. Here "expo" refers to "exponentially" and $\tilde{n}_{i} \equiv n_{i}-\frac{1}{2}$.

\begin{tabular}{lccc}
\hline \hline Properties & $U(1)$ liquid & $\mathbb{Z}_{2}$ liquid & Ferromagnet \\
\hline Spectrum & Gapless & Gapped & Gapped \\
Low- $T C_{v}$ & Power law & Activated & Activated \\
$\left\langle\tilde{n}_{i} \tilde{n}_{j}\right\rangle$ & Power law & Expo decay & Expo decay \\
$\left\langle b_{i}^{\dagger} b_{j}\right\rangle$ & Expo decay & Expo decay & Constant \\
\hline \hline
\end{tabular}

as $t_{1} / V$ increases inside the Coulomb liquid, the spinon gap monotonically decreases, and the interaction $t_{2}$ could lower the spinon pairing energy and overcome the reduced twospinon gap, leading to the $\mathbb{Z}_{2}$ liquid state. However, there is an alternative explanation for the behavior of the specific heat and it is the formation of a charge density wave (CDW). However, stabilizing a CDW would require longer range density-density interactions only generated at high orders in perturbation theory so we consider it less likely. In Ref. [50], we provide more discussion about the detailed features of the specific heat in Fig. 3 and this possible alternative explanation.

As listed in Table I, another important distinction between different quantum phases lies in the spatial dependence of correlation functions. Here we numerically measure the density-density and the boson-boson correlators that are defined as

$$
C_{n}(\boldsymbol{r}) \equiv\left\langle\left(n_{i}-\frac{1}{2}\right)\left(n_{j}-\frac{1}{2}\right)\right\rangle, \quad C_{b}(\boldsymbol{r}) \equiv\left\langle b_{i}^{\dagger} b_{j}\right\rangle,
$$

where $\boldsymbol{r}$ is the spatial separation between sites $i$ and $j$. In the spin language, $C_{n}$ corresponds to the $S^{z}-S^{z}$ correlator, while $C_{b}$ corresponds to the $S^{+}-S^{-}$correlator.

We compare the correlations of the Coulomb liquid and those of the ferromagnetic state. As we depict in Fig. 4, the boson density correlators for the points 1 and 2 decay as

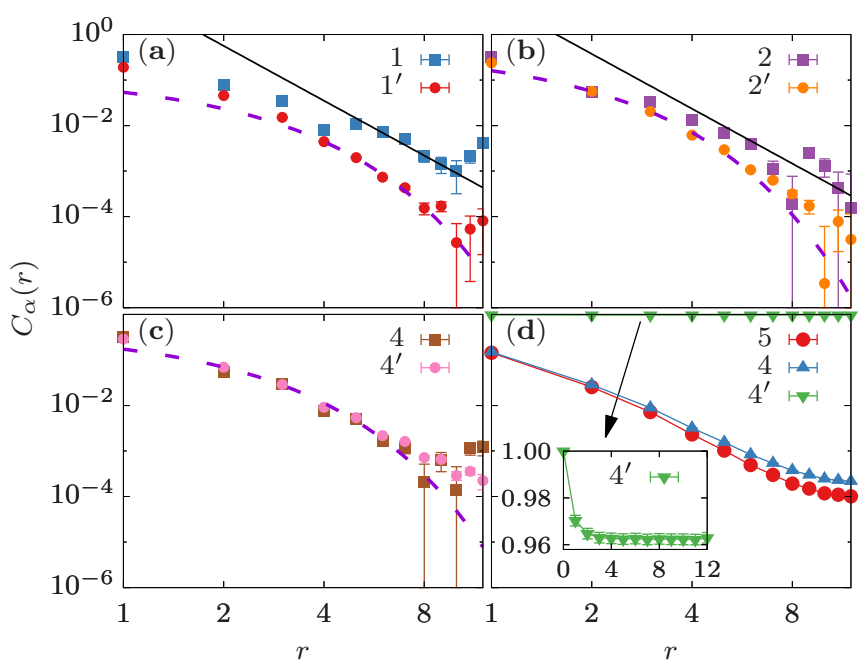

FIG. 4. Panels (a), (b), and (c) show (equal-time) density correlators $C_{n}$ for the given parameter points in the figures, and panel (d) lists the boson-boson correlators $C_{b}$. For $C_{n}$, we have taken the absolute value. The solid (dashed) line refers to the behavior of a $1 / r^{4}$ (exponential) decaying. These data are obtained with the system size $L=12$ and the temperature $T / V=1 / 1000$. a $1 / r^{4}$ power law with the distance, and the boson-boson correlators decay exponentially. This is consistent with the prediction from the Coulomb liquid in which the density correlator at long distances and low energies $[15,16]$ is mapped to the $U(1)$ gauge photon modes [56] and the boson-boson correlator reflects the gapped fractionalized (spinon) quasiparticles [57,58]. In contrast, for the point $4^{\prime}$ inside the ferromagnetic state, the boson density correlator decays exponentially, and the boson-boson correlator saturates to a constant since the system develops the order in $\langle b\rangle$ by breaking the global $\mathbb{Z}_{2}$ symmetry and simultaneously leads to a gap for the density correlator.

For the $\mathbb{Z}_{2}$ liquid, all correlators decay exponentially with the spatial separations. We find the boson-boson correlation does indeed decay exponentially. For the density correlators, despite the thermodynamic gap, we were unable to show more convincingly the exponetially decaying behavior due to the finite system size and the tiny energy gap. To resolve this, one may need even larger system sizes to carry out the simulation in the future.

Discussion. Combining the analytical and numerical analysis, we determine the phase diagram in the sign-problem-free region. When $t_{1}$ and $t_{2}$ are small, a $U(1)$ Coulomb liquid is stable with emergent $U(1)$ gauge structure. As $t_{1}$ or $t_{2}$ increases, exceeding their critical values, the system enter a ferromagnetic phase. However, due to the spinon interaction induced by the pairing term, a $\mathbb{Z}_{2}$ spin liquid is also a possible choice via the condensation of spinon pairs, and thermodynamic evidence is found to support this.

As for the physical realization, the solid-state realization has been proposed for dipole-octupole doublets that have a XYZ model interaction [7,8,38]. Several Nd-based [29-37] and Sm-based [59] pyrochlore magnets [60] were proposed to realize the dipole-octupole doublets, though most of them support magnetic orders with mixed dipolar and octupolar nature [29-37]. The known spin liquid candidate is the Ce-based pyrochlore $\mathrm{Ce}_{2} \mathrm{Sn}_{2} \mathrm{O}_{7}$ and $\mathrm{Ce}_{2} \mathrm{Zr}_{2} \mathrm{O}_{7}$ where the $\mathrm{Ce}^{3+}$ ion gives a dipole-octupole doublet [38-43]. Besides the solid-state context, the cold atoms on optical lattices are used to realize exotic quantum models. In a previous proposal, Ref. [49] has designed a ring exchange interaction for the boson gases via a Raman transition to "molecular" states on optical lattices to simulate the $U(1)$ lattice gauge theory, where this Raman coupling has the form $\phi^{\dagger} b_{i} b_{j}$ and $\phi$ refers to the "molecular" state. Recently, the cold alkali atoms stored in optical lattices or magnetic trap arrays were proposed to realize a broad class of spin-1/2 models including the XYZ model by admixing van der Waals interaction between fine-structure split Rydberg states with laser light. Following these early proposals, we suggest two cold-atom setups to realize our paired hardcore boson model. In the first setup, we follow Ref. [49] and propose a resonant coupling of the bosons via a Raman transition to a "molecular" two-particle state. Instead of choosing the original $d$-wave symmetry to simulate the ring exchange in Ref. [49], we propose an $s$-wave symmetry and condense the molecular states $\phi$. Such a design naturally gives rise to an (uniform) hardcore boson pairing term $\left\langle\phi^{\dagger}\right\rangle b_{i} b_{j}$ for a given lattice. For the second setup, one can directly exploit the known results and methods in Ref. [48] and extend to other lattices. 
Acknowledgments. We would like to thank the hospitality of Zhong Wang at IAS Tsinghua where this work is completed, and particularly acknowledge a previous collaboration with Mike Hermele and Xiaoqun Wang for the encouragements. This work is supported by the Ministry of Science and Technology of China Grants No. 2016YFA0300500, No. 2018YFE0103200, and No. 2016YFA0301001, Thousand-Youth-Talent Program by Shanghai Municipal
Science and Technology Major Project with Grant No. 2019SHZDZX04, the Research Grants Council of Hong Kong with General Research Fund Grants No. 17303819 and No. 17306520 (C.J.H., C.L.L., G.C.), the National Natural Science Foundation of China under Grant No. 11625522, and the Ministry of Science and Technology of China Grants No. 2016YFA0301604 and No. 2016YFA0301001 (C.J.H., Y.J.D.).
[1] A.Y. Kitaev, Fault-tolerant quantum computation by anyons, Ann. Phys. 303, 2 (2003).

[2] M. A. Levin and X.-G. Wen, String-net condensation: A physical mechanism for topological phases, Phys. Rev. B 71, 045110 (2005).

[3] H. Song and M. Hermele, Space-group symmetry fractionalization in a family of exactly solvable models with $\mathbb{Z}_{2}$ topological order, Phys. Rev. B 91, 014405 (2015).

[4] Z.-C. Gu, Z. Wang, and X.-G. Wen, Lattice model for fermionic toric code, Phys. Rev. B 90, 085140 (2014).

[5] A. Kitaev, Anyons in an exactly solved model and beyond, Ann. Phys. 321, 2 (2006).

[6] G. Jackeli and G. Khaliullin, Mott Insulators in the Strong SpinOrbit Coupling Limit: From Heisenberg to a Quantum Compass and Kitaev models, Phys. Rev. Lett. 102, 017205 (2009).

[7] Y.-P. Huang, G. Chen, and M. Hermele, Quantum Spin Ices and Topological Phases from Dipolar-Octupolar Doublets on the Pyrochlore Lattice, Phys. Rev. Lett. 112, 167203 (2014).

[8] Y.-D. Li, X. Wang, and G. Chen, Hidden multipolar orders of dipole-octupole doublets on a triangular lattice, Phys. Rev. B 94, 201114(R) (2016).

[9] M. Hermele, M. P. A. Fisher, and L. Balents, Pyrochlore photons: The $U(1)$ spin liquid in a $S=\frac{1}{2}$ three-dimensional frustrated magnet, Phys. Rev. B 69, 064404 (2004).

[10] C. L. Henley, The Coulomb phase in frustrated systems, Annu. Rev. Condens. Matter Phys. 1, 179 (2010).

[11] H. R. Molavian, M. J. P. Gingras, and B. Canals, Dynamically Induced Frustration as a Route to a Quantum Spin Ice State in $\mathrm{Tb}_{2} \mathrm{Ti}_{2} \mathrm{O}_{7}$ Via Virtual Crystal Field Excitations and Quantum Many-Body Effects, Phys. Rev. Lett. 98, 157204 (2007).

[12] K. A. Ross, L. Savary, B. D. Gaulin, and L. Balents, Quantum Excitations in Quantum Spin Ice, Phys. Rev. X 1, 021002 (2011).

[13] Z. Hao, A. G. R. Day, and M. J. P. Gingras, Bosonic many-body theory of quantum spin ice, Phys. Rev. B 90, 214430 (2014).

[14] S.-B. Lee, S. Onoda, and L. Balents, Generic quantum spin ice, Phys. Rev. B 86, 104412 (2012).

[15] L. Savary and L. Balents, Coulombic Quantum Liquids in Spin1/2 Pyrochlores, Phys. Rev. Lett. 108, 037202 (2012).

[16] O. Benton, O. Sikora, and N. Shannon, Seeing the light: Experimental signatures of emergent electromagnetism in a quantum spin ice, Phys. Rev. B 86, 075154 (2012).

[17] Y. Wan and O. Tchernyshyov, Quantum Strings in Quantum Spin Ice, Phys. Rev. Lett. 108, 247210 (2012).

[18] R. Sibille, N. Gauthier, H. Yan, M. Ciomaga Hatnean, J. Ollivier, B. Winn, U. Filges, G. Balakrishnan, M. Kenzelmann, N. Shannon, and T. Fennell, Experimental signatures of emergent quantum electrodynamics in $\mathrm{Pr}_{2} \mathrm{Hf}_{2} \mathrm{O}_{7}$, Nat. Phys. 14, 711 (2018).
[19] G. Chen, "Magnetic monopole" condensation of the pyrochlore ice $\mathrm{U}(1)$ quantum spin liquid: Application to $\operatorname{Pr}_{2} \mathrm{Ir}_{2} \mathrm{O}_{7}$ and $\mathrm{Yb}_{2} \mathrm{Ti}_{2} \mathrm{O}_{7}$, Phys. Rev. B 94, 205107 (2016).

[20] L. Savary and L. Balents, Disorder-Induced Quantum Spin Liquid in Spin Ice Pyrochlores, Phys. Rev. Lett. 118, 087203 (2017).

[21] J.-J. Wen, S. M. Koohpayeh, K. A. Ross, B. A. Trump, T. M. McQueen, K. Kimura, S. Nakatsuji, Y. Qiu, D. M. Pajerowski, J. R. D. Copley, and C. L. Broholm, Disordered Route to the Coulomb Quantum Spin Liquid: Random Transverse Fields on Spin Ice in $\operatorname{Pr}_{2} \mathrm{Zr}_{2} \mathrm{O}_{7}$, Phys. Rev. Lett. 118, 107206 (2017).

[22] É. Lantagne-Hurtubise, S. Bhattacharjee, and R. Moessner, Electric field control of emergent electrodynamics in quantum spin ice, Phys. Rev. B 96, 125145 (2017).

[23] G. Chen, H.-Y. Kee, and Y. B. Kim, Fractionalized Charge Excitations in a Spin Liquid on Partially Filled Pyrochlore Lattices, Phys. Rev. Lett. 113, 197202 (2014).

[24] G. Chen and P. A. Lee, Emergent orbitals in the cluster Mott insulator on a breathing kagome lattice, Phys. Rev. B 97, 035124 (2018).

[25] G. Chen, H.-Y. Kee, and Y. B. Kim, Cluster Mott insulators and two Curie-Weiss regimes on an anisotropic kagome lattice, Phys. Rev. B 93, 245134 (2016).

[26] J.-P. Lv, G. Chen, Y. Deng, and Z. Y. Meng, Coulomb Liquid Phases of Bosonic Cluster Mott Insulators on a Pyrochlore Lattice, Phys. Rev. Lett. 115, 037202 (2015).

[27] J. Carrasquilla, G. Chen, and R. G. Melko, Tripartite entangled plaquette state in a cluster magnet, Phys. Rev. B 96, 054405 (2017).

[28] J. Carrasquilla, Z. Hao, and R. Melko, A two-dimensional spin liquid in quantum kagome ice, Nat. Commun. 6, 7421 (2015).

[29] M. Ciomaga Hatnean, M. R. Lees, O. A. Petrenko, D. S. Keeble, G. Balakrishnan, M. J. Gutmann, V. V. Klekovkina, and B. Z. Malkin, Structural and magnetic investigations of single-crystalline neodymium zirconate pyrochlore $\mathrm{Nd}_{2} \mathrm{Zr}_{2} \mathrm{O}_{7}$, Phys. Rev. B 91, 174416 (2015).

[30] J. Xu, V. K. Anand, A. K. Bera, M. Frontzek, D. L. Abernathy, N. Casati, K. Siemensmeyer, and B. Lake, Magnetic structure and crystal-field states of the pyrochlore antiferromagnet $\mathrm{Nd}_{2} \mathrm{Zr}_{2} \mathrm{O}_{7}$, Phys. Rev. B 92, 224430 (2015).

[31] V. K. Anand, A. K. Bera, J. Xu, T. Herrmannsdörfer, C. Ritter, and $\mathrm{B}$. Lake, Observation of long-range magnetic ordering in pyrohafnate $\mathrm{Nd}_{2} \mathrm{Hf}_{2} \mathrm{O}_{7}$ : A neutron diffraction study, Phys. Rev. B 92, 184418 (2015).

[32] A. Bertin, P. Dalmas de Réotier, B. Fåk, C. Marin, A. Yaouanc, A. Forget, D. Sheptyakov, B. Frick, C. Ritter, A. Amato, C. Baines, and P. J. C. King, $\mathrm{Nd}_{2} \mathrm{Sn}_{2} \mathrm{O}_{7}$ : An all-in-all-out pyrochlore magnet with no divergence-free field and anomalously 
slow paramagnetic spin dynamics, Phys. Rev. B 92, 144423 (2015).

[33] E. Lhotel, S. Petit, S. Guitteny, O. Florea, M. Ciomaga Hatnean, C. Colin, E. Ressouche, M. R. Lees, and G. Balakrishnan, Fluctuations and All-In-All-Out Ordering in Dipole-Octupole $\mathrm{Nd}_{2} \mathrm{Zr}_{2} \mathrm{O}_{7}$, Phys. Rev. Lett. 115, 197202 (2015).

[34] O. Benton, Quantum origins of moment fragmentation in $\mathrm{Nd}_{2} \mathrm{Zr}_{2} \mathrm{O}_{7}$, Phys. Rev. B 94, 104430 (2016).

[35] J. Xu, C. Balz, C. Baines, H. Luetkens, and B. Lake, Spin dynamics of the ordered dipolar-octupolar pseudospin- $\frac{1}{2}$ pyrochlore $\mathrm{Nd}_{2} \mathrm{Zr}_{2} \mathrm{O}_{7}$ probed by muon spin relaxation, Phys. Rev. B 94, 064425 (2016).

[36] V. K. Anand, D. L. Abernathy, D. T. Adroja, A. D. Hillier, P. K. Biswas, and B. Lake, Muon spin relaxation and inelastic neutron scattering investigations of the all-in/all-out antiferromagnet $\mathrm{Nd}_{2} \mathrm{Hf}_{2} \mathrm{O}_{7}$, Phys. Rev. B 95, 224420 (2017).

[37] P. Dalmas de Réotier, A. Yaouanc, A. Maisuradze, A. Bertin, P. J. Baker, A. D. Hillier, and A. Forget, Slow spin tunneling in the paramagnetic phase of the pyrochlore $\mathrm{Nd}_{2} \mathrm{Sn}_{2} \mathrm{O}_{7}$, Phys. Rev. B 95, 134420 (2017).

[38] Y.-D. Li and G. Chen, Symmetry enriched U(1) topological orders for dipole-octupole doublets on a pyrochlore lattice, Phys. Rev. B 95, 041106(R) (2017).

[39] R. Sibille, E. Lhotel, V. Pomjakushin, C. Baines, T. Fennell, and M. Kenzelmann, Candidate Quantum Spin Liquid in the $\mathrm{Ce}^{3+}$ Pyrochlore Stannate $\mathrm{Ce}_{2} \mathrm{Sn}_{2} \mathrm{O}_{7}$, Phys. Rev. Lett. 115, 097202 (2015).

[40] X.-P. Yao, Y.-D. Li, and G. Chen, Pyrochlore U(1) spin liquid of mixed-symmetry enrichments in magnetic fields, Phys. Rev. Research 2, 013334 (2020).

[41] B. Gao, T. Chen, D. W. Tam, C.-L. Huang, K. Sasmal, D. T. Adroja, F. Ye, H. Cao, G. Sala, M. B. Stone et al., Experimental signatures of a three-dimensional quantum spin liquid in effective spin-1/2 $\mathrm{Ce}_{2} \mathrm{Zr}_{2} \mathrm{O}_{7}$ pyrochlore, Nat. Phys. 15, 1052 (2019).

[42] J. Gaudet, E. M. Smith, J. Dudemaine, J. Beare, C. R. C. Buhariwalla, N. P. Butch, M. B. Stone, A. I. Kolesnikov, Guangyong $\mathrm{Xu}, \mathrm{D}$. R. Yahne, K. A. Ross, C. A. Marjerrison, J. D. Garrett, G. M. Luke, A. D. Bianchi, and B. D. Gaulin, Quantum Spin Ice Dynamics in the Dipole-Octupole Pyrochlore Magnet $\mathrm{Ce}_{2} \mathrm{Zr}_{2} \mathrm{O}_{7}$, Phys. Rev. Lett. 122, 187201 (2019).

[43] R. Sibille, N. Gauthier, E. Lhotel, V. Pore, V. Pomjakushin, R. A. Ewings, T. G. Perring, J. Ollivier, A. Wildes, C. Ritter et al., A quantum liquid of magnetic octupoles on the pyrochlore lattice, Nat. Phys. 16, 546 (2020).

[44] A. Banerjee, S. V. Isakov, K. Damle, and Y. B. Kim, Unusual Liquid State of Hard-Core Bosons on the Pyrochlore Lattice, Phys. Rev. Lett. 100, 047208 (2008).
[45] S. Onoda and Y. Tanaka, Quantum Melting of Spin Ice: Emergent Cooperative Quadrupole and Chirality, Phys. Rev. Lett. 105, 047201 (2010).

[46] N. Shannon, O. Sikora, F. Pollmann, K. Penc, and P. Fulde, Quantum Ice: A Quantum Monte Carlo Study, Phys. Rev. Lett. 108, 067204 (2012).

[47] Y. Kato and S. Onoda, Numerical Evidence of Quantum Melting of Spin Ice: Quantum-To-Classical Crossover, Phys. Rev. Lett. 115, 077202 (2015).

[48] A. W. Glaetzle, M. Dalmonte, R. Nath, C. Gross, I. Bloch, and P. Zoller, Designing Frustrated Quantum Magnets with LaserDressed Rydberg Atoms, Phys. Rev. Lett. 114, 173002 (2015).

[49] H. P. Büchler, M. Hermele, S. D. Huber, Matthew P. A. Fisher, and P. Zoller, Atomic Quantum Simulator for Lattice Gauge Theories and Ring Exchange Models, Phys. Rev. Lett. 95, 040402 (2005).

[50] See Supplemental Material at http://link.aps.org/supplemental/ 10.1103/PhysRevResearch.2.042022 for detailed information about $\mathrm{ABC}$

[51] S. T. Bramwell and M. J. P. Gingras, Spin ice state in frustrated magnetic pyrochlore materials, Science 294, 1495 (2001).

[52] C. Castelnovo, R. Moessner, and S. L. Sondhi, Magnetic monopoles in spin ice, Nature (London) 451, 42 (2008).

[53] M. J. P. Gingras and P. A. McClarty, Quantum spin ice: A search for gapless quantum spin liquids in pyrochlore magnets, Rep. Prog. Phys. 77, 056501 (2014).

[54] N. V. Prokof'ev, B. V. Svistunov, and I. S. Tupitsyn, Exact, complete, and universal continuous-time worldline Monte Carlo approach to the statistics of discrete quantum systems, J. Exp. Theor. Phys. 87, 310 (1998).

[55] N. V. Prokof'ev, B. V. Svistunov, and I. S. Tupitsyn, Worm algorithm in quantum Monte Carlo simulations, Phys. Lett. A 238, 253 (1998).

[56] It was recently realized in Ref. [58] that at higher energies the density correlator would include the magnetic monopole contribution.

[57] G. Chen, Spectral periodicity of the spinon continuum in quantum spin ice, Phys. Rev. B 96, 085136 (2017).

[58] G. Chen, Dirac's "magnetic monopoles" in pyrochlore ice $U(1)$ spin liquids: Spectrum and classification, Phys. Rev. B 96, 195127 (2017).

[59] C. Mauws, A. M. Hallas, G. Sala, A. A. Aczel, P. M. Sarte, J. Gaudet, D. Ziat, J. A. Quilliam, J. A. Lussier, M. Bieringer, H. D. Zhou, A. Wildes, M. B. Stone, D. Abernathy, G. M. Luke, B. D. Gaulin, and C. R. Wiebe, Dipolar-octupolar Ising antiferromagnetism in $\mathrm{Sm}_{2} \mathrm{Ti}_{2} \mathrm{O}_{7}$ : A moment fragmentation candidate, Phys. Rev. B 98, 100401(R) (2018).

[60] J. S. Gardner, M. J. P. Gingras, and J. E. Greedan, Magnetic pyrochlore oxides, Rev. Mod. Phys. 82, 53 (2010). 